\title{
IMPROVEMENT OF THE CAUCHY INTEGRAL METHOD FOR THE STRESSES AND DISPLACEMENTS AROUND A DEEPLY-BURIED NON-CIRCULAR TUNNEL
}

\author{
Yulin Zhou, Aizhong Lu, Hui Cai, Yaocai Ma \\ North China Electric Power University, Institute of Hydroelectric and Geotechnical Engineering, Beijing, China \\ Corresponding author: Aizhong Lu,lvaizhong@ncepu.edu.cn
}

\begin{abstract}
For a deeply-buried non-circular tunnel, abnormal stress and displacement arise in the region far away from the excavation boundary when the Cauchy integral method is applied. It is found that the calculation error is induced by a polynomial in the analytical function. An improved method is proposed by expanding the polynomial as a power series. The improved method is suitable for calculating the stress and displacement in the whole surrounding rock. The results obtained by the improved method are in good agreement with the numerical results of ANSYS, which proves the effectiveness of the proposed method.
\end{abstract}

Keywords: non-circular tunnel, Cauchy integral method, stress and displacement, abnormal calculations, improved method

\section{Introduction}

In the early studies on stresses and displacements around a deeply-buried tunnel with simple shape (circular or oval), some scholars simplified it as the problem of an infinite elastic plane with a hole, and the classical solution to the problem could be widely applied in the design of tunnels (Greenspan, 1944; Inglis, 1913; Terzaghi and Richart, 1952). For a complex-shaped hole, the complex variable method presented by Muskhelishvili (1963) is especially suitable for solving the problem. By using conformal mapping, the area outside the hole in the physical plane ( $z$-plane) can be mapped to the area outside or inside the unit circle in the image plane $(\zeta$-plane), and the problem is reduced to the problem of solving complex potential functions $\varphi(\zeta)$ and $\psi(\zeta)$. The complex potential functions can be obtained by the Cauchy integral of stress or displacement boundary conditions, then substitution of them into the expressions of stress and displacement yields the stress components and displacement components.

Since the Cauchy integral method can efficiently solve boundary value problems of elasticity, many scholars adopted the method to solve stresses and displacements around the tunnel. For an infinite plate subjected to uniform tensile stress containing a rectangular hole with fillet, Motok (1997) obtained complex potential functions and analyzed the influence of curvature radius of the fillet on the stress concentration factor. For an infinite plate with a rectangular hole subjected to uniaxial stress at infinity, Lei et al. (2001) introduced a new correction factor to improve the accuracy of the mapping function and obtained the distribution of stress and displacement at the edge of the hole. Exadaktylos and Stavropoulou (2002) calculated stresses and displacements at the edge of a deeply-buried semi-circular tunnel under arbitrary in-situ stress. For an infinite plate subjected to arbitrary biaxial loadings at infinity containing a polygonal hole, Sharma (2012) obtained complex potential functions and stress at the edge of the hole, and discussed the effect of the hole geometry and loading pattern on the stress concentration factor. Shi and Gao (2014) determined parameters of the mapping function of the horseshoe-shaped tunnel through the composite optimization technology, and obtained the stress and displacement at 
the edge of the hole under arbitrary in-situ stress. For a square tunnel under arbitrary in-situ stress, Zhao and Yang (2015) obtained the stress at the edge of the hole under different lateral pressure coefficients. For an infinite plate with a hypotrochoidal hole subjected to uniaxial (or biaxial) stress at infinity, Sharma (2016) calculated the stress and stress intensity factor at the edge of the hole, and analyzed the effect of the hole geometry and loading conditions on the stress.

As mentioned before, for differently shaped tunnels (holes) without support subjected to different loads, the complex potential functions can be solved through the Cauchy integral method, and the accurate stress and displacement at the edge of the hole can be calculated using the complex potential functions. However, the stress and displacement outside the hole are not involved. It can be seen from the research of this paper: when the complex potential functions obtained by the traditional Cauchy integral method are applied to the calculation of stress and displacement around the complex-shaped hole, solutions with enough accuracy at the edge of the hole can be obtained. However, as the distance from the calculated position to the hole edge becomes larger, the accuracy of the calculation of stresses and displacements becomes increasingly poor. When the distance exceeds a certain value, there are violent fluctuations of the stress curve and displacement curve, that is, the stress and displacement at the area outside the hole obtained by the traditional Cauchy integral method are abnormal (as shown in Fig. 6). If these calculation results are applied to an engineering design, it is bound to bring wrong conclusions. For example, the displacement outside the hole is often used in displacement back analysis. Compared with the general numerical method, the Cauchy integral method is used to calculate the displacement of the surrounding rock, which can avoid solving the large sparse matrix and greatly improve the calculation efficiency of displacement back analysis (Jiao et al., 2009). However, if the displacement outside the hole calculated by the Cauchy integral method is abnormal, the in-situ stress and mechanical parameters of the surrounding rock calculated by the displacement back analysis will be inconsistent with the actual situation. Therefore, safety of the tunnel engineering construction cannot be correctly judged (Fakhimi et al., 2004; Kodama et al., 2013).

In this paper, the causes of abnormal calculation at the area outside the hole are analyzed, and the traditional Cauchy integral method is improved. A correct and reliable calculation method is given, which can solve the problem of abnormal calculation.

\section{The process and analysis of the traditional Cauchy integral method}

\subsection{Basic assumptions}

In this paper, a deeply-buried tunnel with a symmetry axis ( $x$-axis) is studied, as shown in Fig. 1. Under the in-situ stress $\sigma_{x}^{\infty}=P$ and $\sigma_{y}^{\infty}=Q$, the surrounding rock is assumed to be in an elastic state and the strain along the axis of the tunnel is equal to 0 , then the mechanical analysis of surrounding rock is simplified to the plane strain problem.

\subsection{Mapping function}

To solve the problem given in Fig. 1, the area outside the tunnel in the $z$-plane is mapped to the outer area of the unit circle in the $\zeta$-plane by a conformal mapping function (Fig. 2), and the solving equations expressed by the variable $z$ are transformed into the equations expressed by the variable $\zeta$. The mapping function is (Muskhelishvili, 1963)

$$
z=\omega(\zeta)=R\left(\zeta+\sum_{k=0}^{n} C_{k} \zeta^{-k}\right)
$$




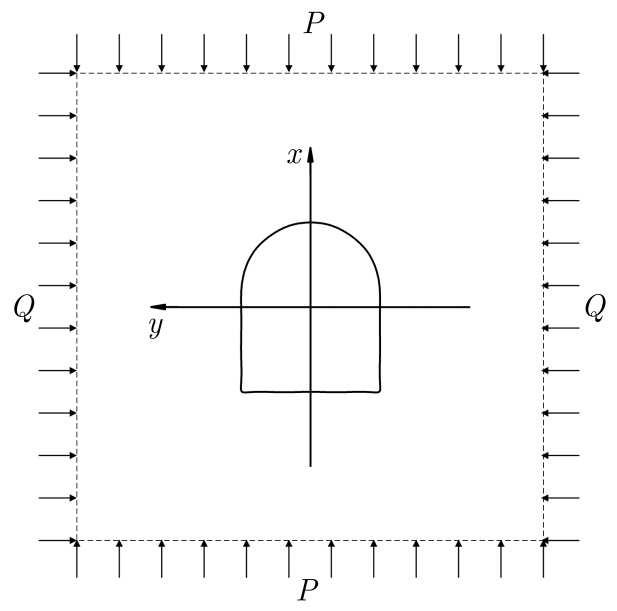

Fig. 1. Deeply-buried tunnel under the in-situ stress
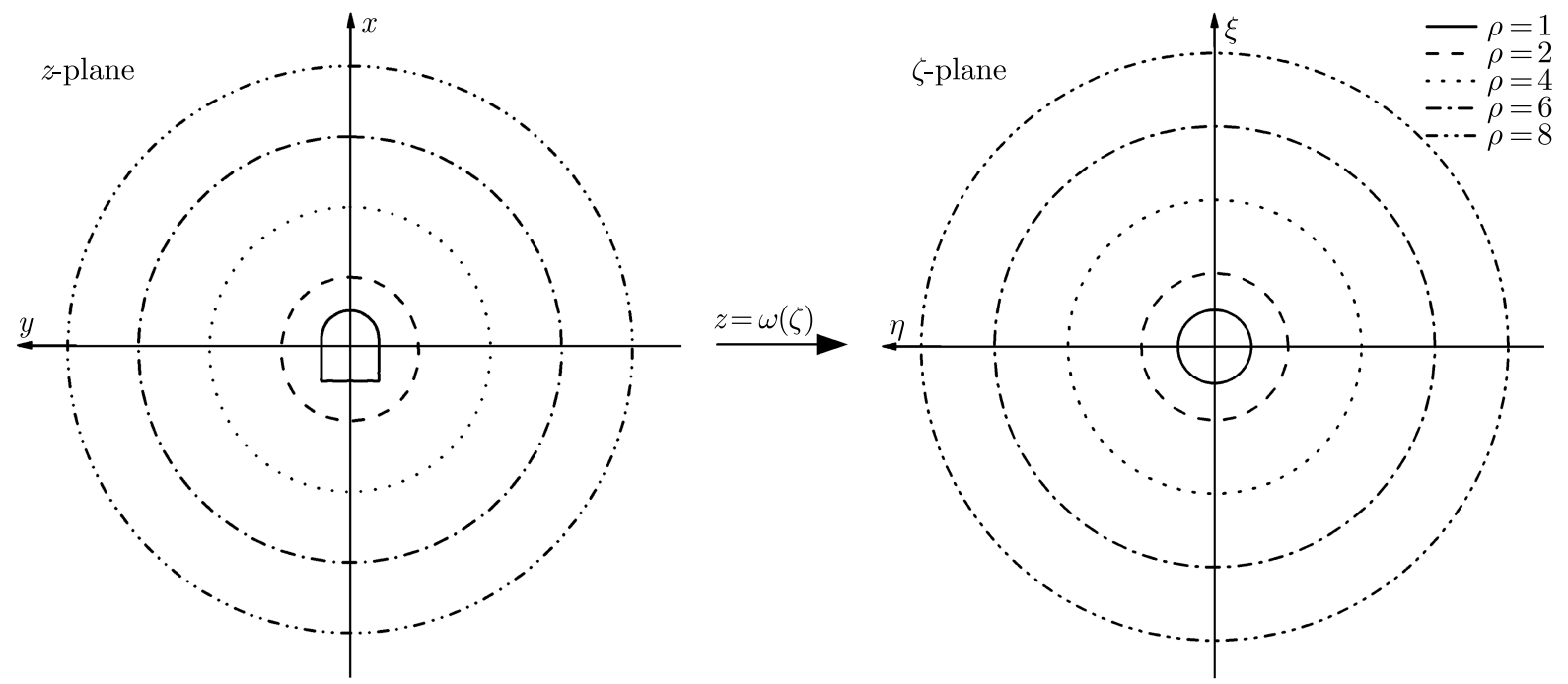

Fig. 2. The area outside the tunnel in the $z$-plane is mapped to the outer area of the unit circle in the $\zeta$-plane

where $R$ is a positive real number reflecting the size of the tunnel. $C_{k}$ are constants reflecting the cross-section shape of the tunnel, when the $x$-axis is the symmetry axis of the tunnel, $C_{k}$ are real numbers. $\zeta=\rho \mathrm{e}^{\mathrm{i} \theta}, \rho$ and $\theta$ are the polar diameter and polar angle of the $\zeta$-plane, respectively. $n$ is the number of terms of the coefficients in the mapping function. The size of $n$ depends on the complexity of the tunnel shape. The more complex the tunnel shape, the greater the value of $n$.

The graphs in the $z$-plane can be mapped to circles with $\rho=1,2,4,6,8$ in the $\zeta$-plane, as shown in Fig. 2. $\rho=1$ corresponds to the edge of the tunnel, and $\rho>1$ corresponds to a family of coordinate lines in the $z$-plane. These coordinate lines are not circular, but with an increase of $\rho$, the corresponding coordinate lines tend to be circles, which can be seen clearly from Eq. (2.1) and Fig. 2.

\subsection{Expressions of the stress and displacement expressed by analytical functions}

According to the complex variable method presented by Muskhelishvili (1963), combinations of stress and displacement are expressed by the analytical functions 


$$
\begin{aligned}
& \sigma_{\rho}+\sigma_{\theta}=4 \operatorname{Re}\left[\frac{\varphi^{\prime}(\zeta)}{\omega^{\prime}(\zeta)}\right] \\
& \sigma_{\rho}-\sigma_{\theta}+2 \mathrm{i} \tau_{\rho \theta}=\frac{2 \zeta^{2}}{\rho^{2}} \frac{1}{\overline{\omega^{\prime}(\zeta)}}\left\{\overline{\omega(\zeta)} \frac{\varphi^{\prime \prime}(\zeta) \omega^{\prime}(\zeta)-\varphi^{\prime}(\zeta) \omega^{\prime \prime}(\zeta)}{\left[\omega^{\prime}(\zeta)\right]^{2}}+\psi^{\prime}(\zeta)\right\} \\
& 2 G(u+\mathrm{i} v)=\kappa \varphi_{0}(\zeta)-\frac{\omega(\zeta)}{\overline{\omega^{\prime}(\zeta)}} \overline{\varphi_{0}^{\prime}(\zeta)}-\overline{\psi_{0}(\zeta)}
\end{aligned}
$$

where $\sigma_{\rho}, \sigma_{\theta}$ and $\tau_{\rho} \theta$ are the normal, tangential and shear stress in the orthogonal curvilinear coordinate (in $z$-plane), respectively. $u$ and $v$ are displacements in the $x$ and $y$ directions (in $z$-plane), respectively, i.e., vertical and horizontal displacement, they represent the displacements resulting from the excavation of the tunnel. $G$ is the shear modulus of the material, $G=E /(2+2 \mu)$, where $E$ and $\mu$ are the elastic modulus and Poisson's ratio of the material, respectively. For the plane strain problem, $\kappa=3-4 \mu$. For an unsupported tunnel, the expressions of the analytical functions $\varphi(\zeta)$ and $\psi(\zeta)$ are (Muskhelishvili, 1963)

$$
\varphi(\zeta)=B \omega(\zeta)+\varphi_{0}(\zeta) \quad \psi(\zeta)=\left(B^{\prime}+\mathrm{i} C^{\prime}\right) \omega(\zeta)+\psi_{0}(\zeta)
$$

where $B, B^{\prime}, C^{\prime}$ are constants, $B=(P+Q) / 4, B^{\prime}=(Q-P) / 2, C^{\prime}=0 ; \varphi_{0}(\zeta)$ and $\psi_{0}(\zeta)$ are single-valued analytical functions in the exterior of the unit circle, and their general expressions are written as the following series (Muskhelishvili, 1963)

$$
\varphi_{0}(\zeta)=\sum_{j=1}^{\infty} a_{j} \zeta^{-j} \quad \psi_{0}(\zeta)=\sum_{j=1}^{\infty} b_{j} \zeta^{-j}
$$

where $a_{j}, b_{j}$ are complex constants to be solved.

It can be seen from the following derivation process: $\varphi_{0}(\zeta)$ obtained by the Cauchy integral method is a finite series, and the maximum value of $j$ is equal to $n$ ( $n$ is the number of terms of the coefficients in the mapping function, i.e., Eq. $(2.1))$, but $\psi_{0}(\zeta)$ is a rational fraction about $\zeta$ (Muskhelishvili, 1963). If $\psi_{0}(\zeta)$ is expanded as a power series, it must be an infinite series.

\subsection{Analytical functions and solutions of stress and displacement components}

$\varphi(\zeta)$ and $\psi(\zeta)$ can be obtained by the stress boundary condition as

$$
\varphi(\sigma)+\frac{\omega(\sigma)}{\overline{\omega^{\prime}(\sigma)}} \overline{\varphi^{\prime}(\sigma)}+\overline{\psi(\sigma)}=f(\sigma)
$$

where $\sigma$ is the point at the unit circle in the $\zeta$-plane, $\sigma=\mathrm{e}^{\mathrm{i} \theta}$; when there is no external load at the edge of the tunnel, $f(\sigma)=0$.

At the edge of the tunnel, $\zeta=\sigma$, substituting Eqs. (2.3), into Eq. (2.5) yields

$$
\varphi_{0}(\sigma)+\frac{\omega(\sigma)}{\overline{\omega^{\prime}(\sigma)}} \overline{\varphi_{0}^{\prime}(\sigma)}+\overline{\psi_{0}(\sigma)}=-2 B \omega(\sigma)-\left(B^{\prime}-\mathrm{i} C^{\prime}\right) \overline{\omega(\sigma)}=f_{0}(\sigma)
$$

Substituting $B=(P+Q) / 4, B^{\prime}=(Q-P) / 2, C^{\prime}=0$ into Eq. (2.6) yields

$$
f_{0}(\sigma)=-\frac{P+Q}{2} \omega(\sigma)+\frac{P-Q}{2} \overline{\omega(\sigma)}=-\frac{P}{2}(1+\lambda) \omega(\sigma)+\frac{P}{2}(1-\lambda) \overline{\omega(\sigma)}
$$

where $\lambda$ is the lateral pressure coefficient, $\lambda=Q / P$. 
The equations for solving $\varphi_{0}(\zeta)$ and $\psi_{0}(\zeta)$ are obtained by applying Eq. (2.6) and its conjugate to the Cauchy integral operator, respectively

$$
\begin{aligned}
& \varphi_{0}(\zeta)=\frac{1}{2 \pi \mathrm{i}} \int_{r} \frac{\omega(\sigma)}{\overline{\omega^{\prime}(\sigma)}} \frac{\overline{\varphi_{0}^{\prime}(\sigma)}}{\sigma-\zeta} d \sigma-\frac{1}{2 \pi \mathrm{i}} \int_{r} \frac{f_{0}(\sigma)}{\sigma-\zeta} d \sigma \\
& \psi_{0}(\zeta)=\frac{1}{2 \pi \mathrm{i}} \int_{r} \frac{\overline{\omega(\sigma)}}{\omega^{\prime}(\sigma)} \frac{\varphi_{0}^{\prime}(\sigma)}{\sigma-\zeta} d \sigma-\frac{1}{2 \pi \mathrm{i}} \int_{r} \frac{\overline{f_{0}(\sigma)}}{\sigma-\zeta} d \sigma
\end{aligned}
$$

where $\omega(\zeta) / \overline{\omega^{\prime}}(1 / \zeta)$ can be expressed by the Laurent series as (Muskhelishvili, 1963)

$$
\begin{gathered}
\frac{\omega(\zeta)}{\overline{\omega^{\prime}}(1 / \zeta)}=\frac{C_{n}+C_{n-1} \zeta+\cdots+C_{0} \zeta^{n}+\zeta^{n+1}}{\zeta^{n}\left(1-C_{1} \zeta^{2}-2 C_{2} \zeta^{3}-\cdots-n C_{n} \zeta^{n+1}\right)} \\
=L_{n} \zeta^{-n}+L_{n-1} \zeta^{-n+1}+\cdots+L_{1} \zeta^{-1}+\sum_{k=0}^{\infty} L_{k}^{\prime} \zeta^{k}
\end{gathered}
$$

$L_{k}(k=1, \ldots, n)$ and $L_{k}^{\prime}(k=1, \ldots, \infty)$ are obtained by comparing the coefficients of the same powers of $\zeta$ in both sides of Eq. (2.9). The traditional Cauchy integral solution only needs $L_{k}$, but not $L_{k}^{\prime}$. For different $n$, the expression of $L_{k}$ is

$$
\begin{array}{ll}
n=1 & L_{1}=C_{1} \\
n=2 & L_{1}=C_{1} \quad L_{2}=C_{2} \\
n \geqslant 3 & \left\{\begin{array}{l}
L_{n}=C_{n} \\
L_{n-1}=C_{n-1} \\
L_{n-j+1}=\sum_{k=1}^{j-2}(j-1-k) C_{j-1-k} L_{n-k+1}+C_{n-j+1} \quad j=3, \ldots, n
\end{array}\right.
\end{array}
$$

When $n \leqslant 2, \varphi_{0}(\zeta)$ and $\psi_{0}(\zeta)$ are easily obtained

$$
\begin{aligned}
& \varphi_{0}(\zeta)=\frac{P R}{2}(1-\lambda) \zeta^{-1}-\frac{P R}{2}(1+\lambda)\left(C_{1} \zeta^{-1}+\cdots+C_{n} \zeta^{-n}\right) \\
& \psi_{0}(\zeta)=-\bar{\omega}\left(\frac{1}{\zeta}\right) \frac{\varphi_{0}^{\prime}(\zeta)}{\omega^{\prime}(\zeta)}-\frac{P R}{2}(1+\lambda) \zeta^{-1}+\frac{P R}{2}(1-\lambda)\left(C_{1} \zeta^{-1}+\cdots+C_{n} \zeta^{-n}\right)
\end{aligned}
$$

When $n \geqslant 3$, it can be obtained from Eqs. $(2.4)_{1}$ and (2.9) that

$$
\frac{\omega(\sigma)}{\overline{\omega^{\prime}(\sigma)}} \overline{\varphi_{0}^{\prime}(\sigma)}=S_{1} \sigma^{-1}+S_{2} \sigma^{-2}+\cdots+S_{n-2} \sigma^{-n+2}+\sum_{k=0}^{\infty} S_{k}^{\prime} \sigma^{k}
$$

When $\zeta=\sigma$, substituting Eqs. $(2.4)_{1}$ and $(2,9)$ into Eq. (2.12), then by comparing the coefficients of the same powers of $\sigma$, we have

$$
S_{k}=-\sum_{j=1}^{n-k-1} j \bar{a}_{j} L_{k+1+j} \quad 1 \leqslant k \leqslant n-2
$$

It is obtained by taking the conjugation of Eq. (2.12) that

$$
\frac{\overline{\omega(\sigma)}}{\omega^{\prime}(\sigma)} \varphi_{0}^{\prime}(\sigma)=\bar{S}_{1} \sigma+\bar{S}_{2} \sigma^{2}+\cdots+\bar{S}_{n-2} \sigma^{n-2}+\sum_{k=0}^{\infty} \overline{S^{\prime}}{ }_{k} \sigma^{-k}
$$


The following results are obtained by using the Cauchy integral formula that

$$
\begin{aligned}
& \frac{1}{2 \pi \mathrm{i}} \int_{r} \frac{\omega(\sigma)}{\overline{\omega^{\prime}(\sigma)}} \frac{\overline{\varphi_{0}^{\prime}(\sigma)}}{\sigma-\zeta} d \sigma=-S_{1} \zeta^{-1}-S_{2} \zeta^{-2}-\cdots-S_{n-2} \zeta^{-n+2} \\
& \frac{1}{2 \pi \mathrm{i}} \int_{r} \frac{\overline{\omega(\sigma)}}{\omega^{\prime}(\sigma)} \frac{\varphi_{0}^{\prime}(\sigma)}{\sigma-\zeta} d \sigma=-\sum_{k=0}^{\infty} \overline{{S^{\prime}}}{ }_{k} \zeta^{-k}=-\bar{\omega}\left(\frac{1}{\zeta}\right) \frac{\varphi_{0}^{\prime}(\zeta)}{\omega^{\prime}(\zeta)}+\sum_{k=1}^{n-2} S_{k} \zeta^{k}
\end{aligned}
$$

Substituting Eqs. $(2.4)_{1},(2.13)$ and $(2.15)_{1}$ into Eq. (2.8) $)_{1}$, then by comparing the coefficients of the same powers of $\zeta$, we have

$$
\begin{aligned}
& a_{j}=0 \quad j>n \\
& -a_{n}=2 \Gamma R C_{n} \\
& -a_{n-1}=2 \Gamma R C_{n-1} \\
& -a_{n-2}+L_{n} \bar{a}_{1}=2 \Gamma R C_{n-2} \\
& \vdots \\
& -a_{2}+(n-3) L_{n} \bar{a}_{n-3}+(n-4) L_{n-1} \bar{a}_{n-4}+\cdots+2 L_{5} \bar{a}_{2}+L_{4} \bar{a}_{1}=2 \Gamma R C_{2} \\
& -a_{1}+(n-2) L_{n} \bar{a}_{n-2}+(n-3) L_{n-1} \bar{a}_{n-3}+\cdots+2 L_{4} \bar{a}_{2}+L_{3} \bar{a}_{1}=2 \Gamma R C_{1}+\Gamma^{\prime} R
\end{aligned}
$$

where $\Gamma=P(1+\lambda) / 4, \Gamma^{\prime}=P(\lambda-1) / 2$. For the problem in this paper, $a_{j}$ are the real numbers (Lu and Zhang, 2007). It can be proved by Eq. (2.16). The values of $a_{j}(j=1, \ldots, n)$ are obtained by solving this system of linear equations, i.e., Eq. (2.16). The obtained $\varphi_{0}(\zeta)$ is a finite series $\sum_{j=1}^{n} a_{j} \zeta^{-j}, S_{k}(k=1, \ldots, n-2)$ are obtained by substituting the obtained $a_{j}(j=1, \ldots, n)$ into Eq. (2.13).

Substituting Eq. (2.15) 2 into Eq. $(2.8)_{2}$ yields the expression of $\psi_{0}(\zeta)$

$$
\psi_{0}(\zeta)=-\bar{\omega}\left(\frac{1}{\zeta}\right) \frac{\varphi_{0}^{\prime}(\zeta)}{\omega^{\prime}(\zeta)}+\sum_{k=1}^{n-2} S_{k} \zeta^{k}-\frac{P R}{2}(1+\lambda) \zeta^{-1}+\frac{P R}{2}(1-\lambda) \sum_{j=1}^{n} C_{j} \zeta^{-j}
$$

The first term on the right hand side of Eq. (2.17) is a rational fraction of $\zeta$. If it is expanded as a power series which must be infinite.

$\varphi(\zeta)$ and $\psi(\zeta)$ can be obtained by substituting $\varphi_{0}(\zeta)$ and $\psi_{0}(\zeta)$ into Eq. $(2.3)_{1}$, then substituting $\varphi(\zeta)$ and $\psi(\zeta)$ into Eqs. (2.2) $)_{1,2}$ yields the stress of any point in the surrounding rock. Substituting $\varphi_{0}(\zeta)$ and $\psi_{0}(\zeta)$ into Eq. $(2.2)_{3}$ yields the displacement of any point in the surrounding rock caused by tunnel excavation.

\subsection{Comparison of the analytical and numerical solutions}

For complex-shaped tunnels, the vertical-wall semicircle tunnel (Fig. 1) is taken as an example to illustrate the limitations of the traditional Cauchy integral method. The conformal mapping function in Fig. 1 is as follows

$$
\begin{aligned}
z= & 1.6051\left(\zeta-0.1040+0.0850 \zeta^{-1}+0.0766 \zeta^{-2}-0.0970 \zeta_{-3}+0.0385 \zeta_{-4}\right. \\
& +0.0046 \zeta_{-5}-0.0091 \zeta_{-6}+0.0068 \zeta_{-7}-0.0053 \zeta_{-8}+0.0011 \zeta_{-9}+0.0020 \zeta_{-10} \\
& \left.-0.0036 \zeta_{-11}+0.0013 \zeta_{-12}-0.0001 \zeta_{-13}+0.0001 \zeta_{-14}\right)
\end{aligned}
$$

The in-situ stress and parameters of the surrounding rock are: $P=10.0 \mathrm{MPa}, Q=5.0 \mathrm{MPa}$ and $E=20 \mathrm{GPa}$. The comparison of analytical and numerical solutions of stress and displacement at $\rho=1, \rho=6$ and $\rho=8$ are given in Figs. 4-6, respectively. Due to symmetry of the 
structure and load, only the results on the left side of the axis of symmetry in Fig. 1 are compared, $\theta=0^{\circ}$ corresponds to the point on the $x$-axis above the arch and $\theta=180^{\circ}$ corresponds to the point on the $x$-axis below the hole. The normal stress and tangential stress are positive in compression and negative in tension. The direction of displacement is opposite to the positive and negative directions specified by the coordinate axes.

In this paper, ANSYS is adopted to perform numerical analysis of the tunnel. The calculation model is shown in Fig. 3: the radius of the semi-circular part of the tunnel is about $1.30 \mathrm{~m}$, and the vertical length of the vertical-wall part of the tunnel is about $1.90 \mathrm{~m}$. To simulate the infinite domain, the outer boundary of the model is a square with size $1000 \mathrm{~m} \times 1000 \mathrm{~m}$. The unit of PLANE42 is used to divide the model. When ANSYS is used to calculate the stress, a uniform load of 10.0 MPa is applied to the upper and lower boundaries of the model, and a uniform load of $5.0 \mathrm{MPa}$ is applied to the left and right boundaries of the model. Due to symmetry of the load and structure, the displacement along the $y$-direction on the $x$-axis is equal to 0 . Therefore, the constraints of $y$-direction are applied to the lines on the $x$-axis, and the constraints of $x$-direction are applied to the key points in the lower-left corner and the lower-right corner of the outer boundary of the model (Fig. 3a). When ANSYS is used to calculate the displacement, to simulate the tunnel excavation process, the reverse release load is applied on the unit node of the excavation boundary (Duncan and Dunlop, 1968). It is considered that the displacement on the outer boundary of the model caused by excavation is 0 . Hence, the constraints of $y$-direction are imposed on the upper and lower boundaries of the model, and the constraints of $x$-direction are imposed on the left and right boundaries of the model (Fig. 3b).

(a)

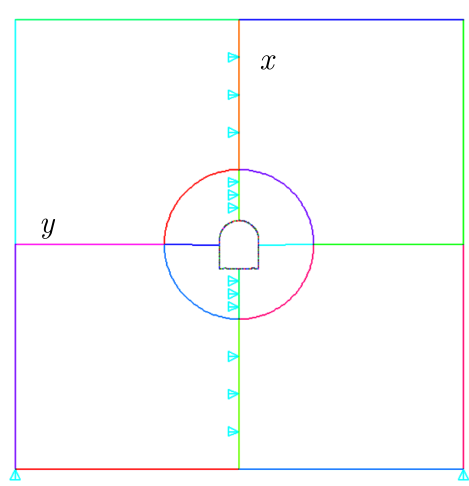

(b)

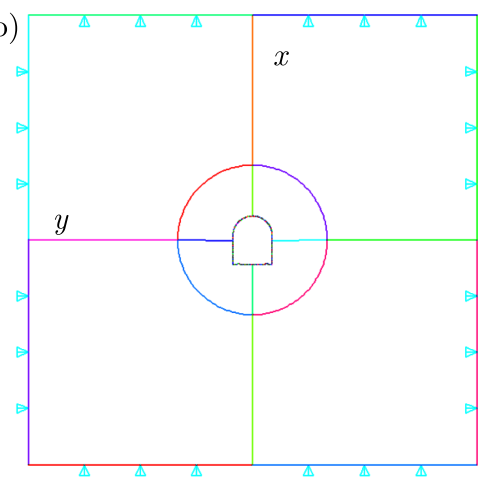

Fig. 3. Constraints applied in: (a) stress calculation, (b) displacement calculation

Since the normal stress and shear stress at the edges of the tunnel are both equal to 0, only the comparison of the tangential stress is given in Fig. 4. It can be seen from Figs. 4-6 that:

The analytical solutions of the tangential stress and displacement at the edges of the tunnel are basically the same as the numerical solutions. The analytical solutions of the stress and displacement at $\rho=6$ begin to fluctuate, which are slightly different from the numerical solutions. However, the analytical solutions of stress and displacement at $\rho=8$ fluctuated violently, which is greatly different from the numerical solution and not in line with the actual situation. It can be seen that the traditional method can solve the accurate stress and displacement at the edge of the tunnel, but the farther from the edge of the tunnel, the worse the calculation accuracy, until the abnormal calculation appears. This is the result of the calculation error. In this paper, all analytical solutions are calculated by Fortran. 
(a)

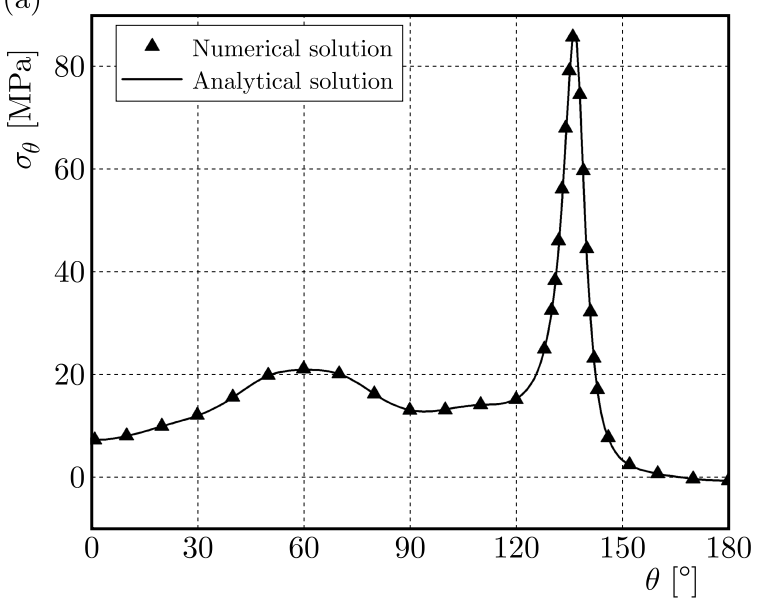

(b)

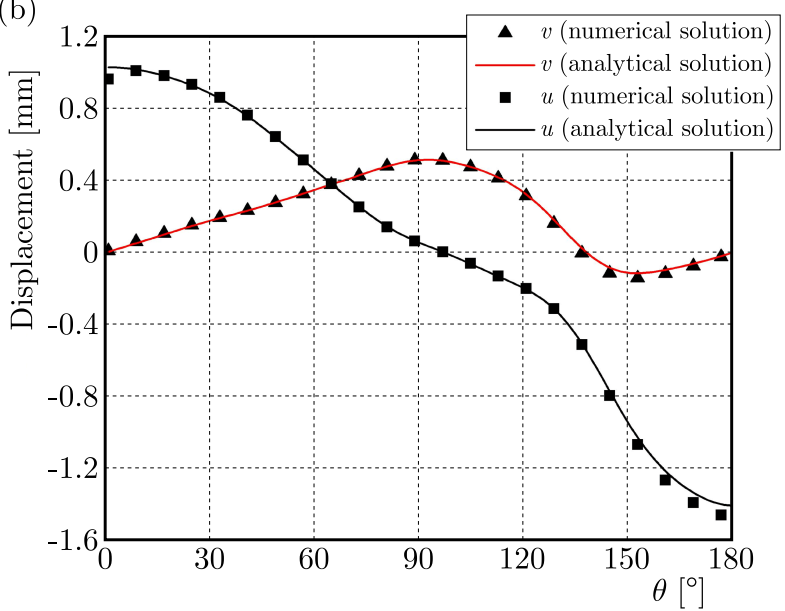

Fig. 4. The contrast between analytical solution obtained by the traditional Cauchy integral method and numerical solution when $\rho=1$ : (a) comparison of tangential stress, (b) comparison of displacement
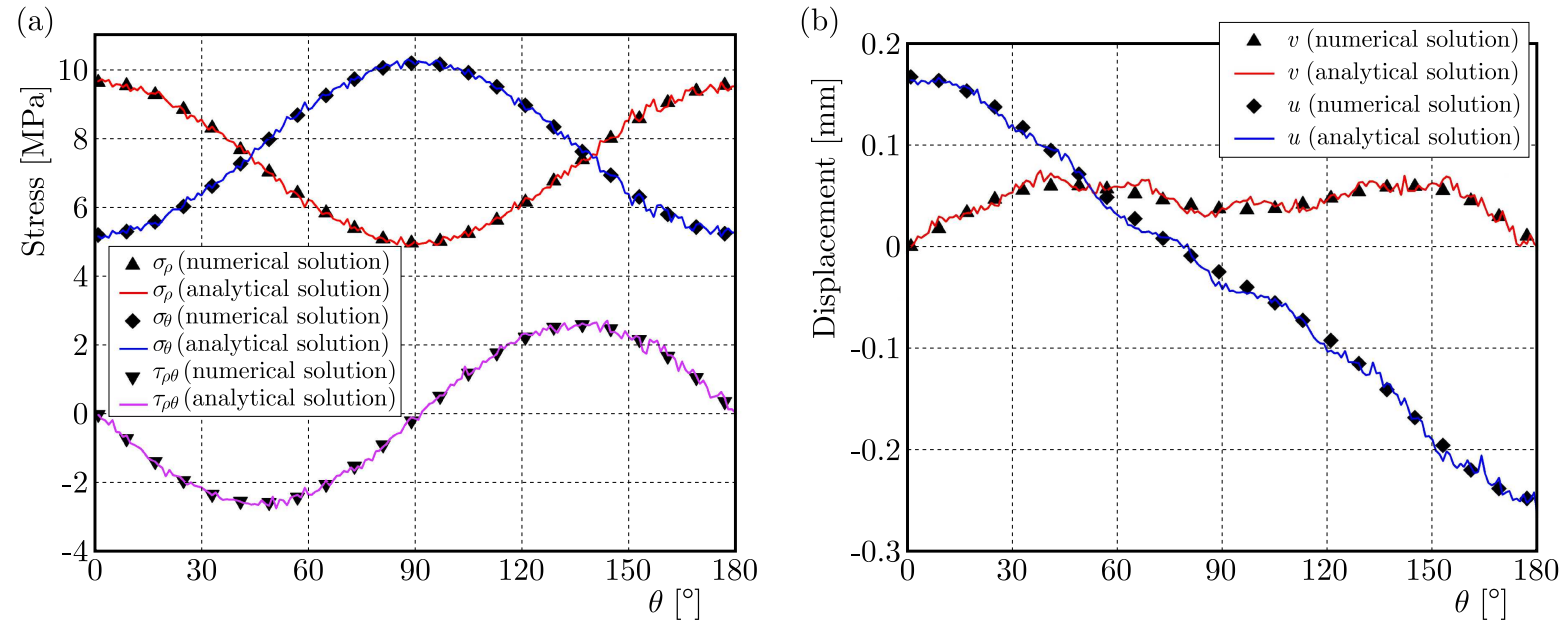

Fig. 5. The contrast between analytical solution obtained by the traditional Cauchy integral method and numerical solution when $\rho=6$ : (a) comparison of stress, (b) comparison of displacement
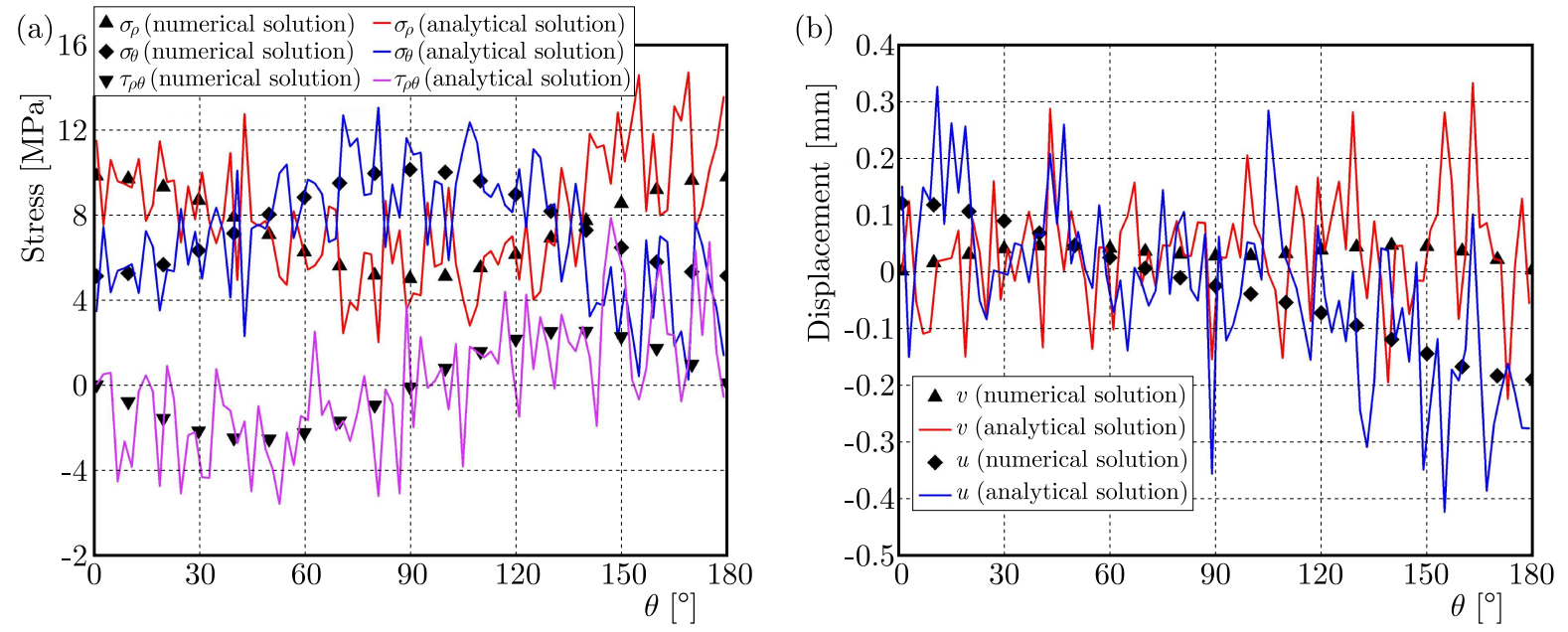

Fig. 6. The contrast between analytical solution obtained by the traditional Cauchy integral method and numerical solution when $\rho=8$ : (a) comparison of stress, (b) comparison of displacement 


\section{Improved Cauchy integral method}

\subsection{Causes of the abnormal calculation obtained by the traditional Cauchy integral method}

Theoretically, since $\psi_{0}(\zeta)$ is an analytical function in the exterior region of the unit circle, $\psi_{0}(\zeta)$ can only be a series of negative power terms about $\zeta$ (Eqs. $\left.(2.4)_{2},(2.15)_{2}\right)$. But it can be seen from Eq. (2.17) that $\sum_{k=1}^{n-2} S_{k} \zeta^{k}$ appears in the obtained $\psi_{0}(\zeta)$. When $n \geqslant 3$, it is a positive power series of $\zeta$ and an analytical function in the interior of the unit circle, not an analytical function in the exterior of the unit circle. This can be clearly explained from the derivation process in Section 2.4.

It can be seen from the analysis of $-\bar{\omega}(1 / \zeta) \varphi_{0}^{\prime}(\zeta) / \omega^{\prime}(\zeta)$ in $\psi_{0}(\zeta)$ that a part of the series of positive power terms about $\zeta$ can be separated from it. Theoretically, it should just cancel out with $\sum_{k=1}^{n-2} S_{k} \zeta^{k}$, but in fact, due to the rounding error (Sauer, 2012), the positive power terms of $-\bar{\omega}(1 / \zeta) \varphi_{0}^{\prime}(\zeta) / \omega^{\prime}(\zeta)$ will not exactly cancel out with $\sum_{k=1}^{n-2} S_{k} \zeta^{k}$ when the computer is processing. The existence of the positive power term of $\zeta$ in $\psi_{0}(\zeta)$ is the main cause of the calculation error. The positive power of $\zeta$ is high when the number of terms of the mapping function coefficient is large (i.e., $\mathrm{n}$ is large), and the value of $|\zeta|(\rho)$ is large at the place far away from the tunnel edge and, therefore, there are some positive high powers of large values in the actual calculation, which leads to a large calculation error and abnormal calculation.

\subsection{The effects of the number of mapping function items on the calculation results}

According to the analysis in Section 3.1, there is no positive power term of $\zeta$ in $\psi_{0}(\zeta)$ when $n \leqslant 2$, thus the calculations of stress and displacement are not abnormal. Then, $n$ (in Eq. (2.18)) is taken as 3, 6 and 12, respectively. The corresponding tunnel shape and the corresponding shape (dotted line) when $\rho=8$ are shown in Fig. 7. The in-situ stress and parameters of the surrounding rock are the same as in Section 2.5.
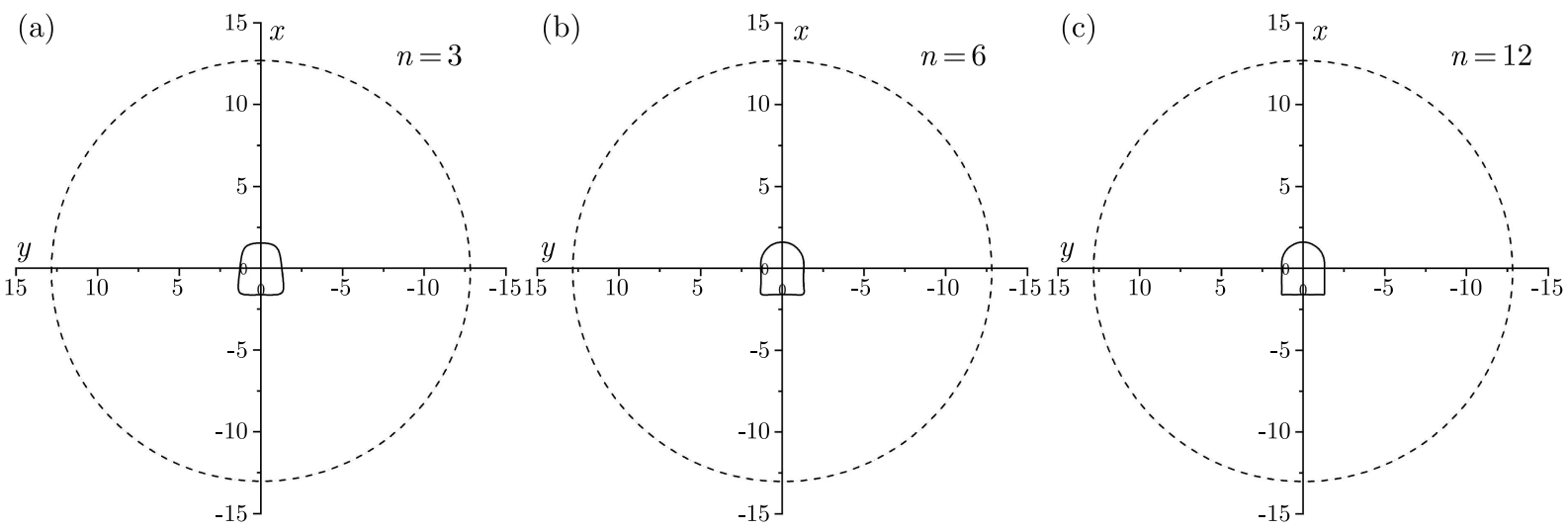

Fig. 7. The corresponding shape when $\rho=8$ and the corresponding tunnel shape for different $n$ :

(a) $n=3$, (b) $n=6$, (c) $n=12$

Figure 8 shows the comparison of analytical and numerical solutions of tangential stress corresponding to different $n$ when $\rho=8$. It can be seen from Fig. 8 that:

When $\rho=8$, the analytical solutions of the tangential stress corresponding to $n \leqslant 6$ are basically the same as the numerical solutions, but the analytical solutions corresponding to $n=12$ fluctuate greatly and differ greatly from the numerical solution, which is not in line with the actual situation. It is concluded that at a position far away from the tunnel edge, when $n$ increases to a certain value, the analytical solutions begin to fluctuate. The fluctuations of the analytical solutions are larger and the calculation accuracy became worse with an increase of $n$. This is consistent with analysis of the calculation error in Section 3.1. 
(a)

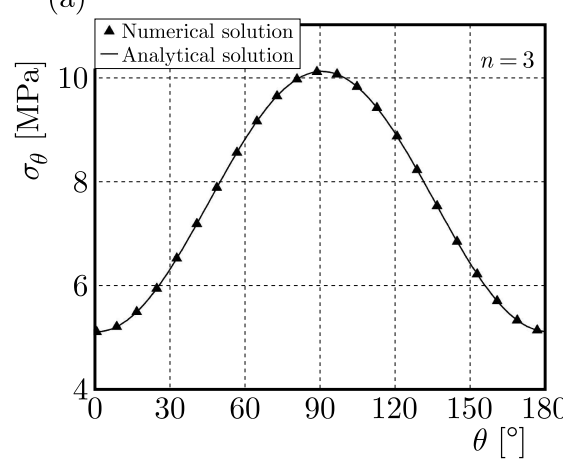

(b)

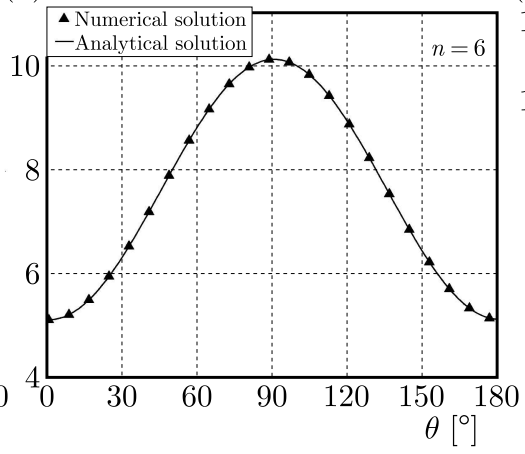

(c)

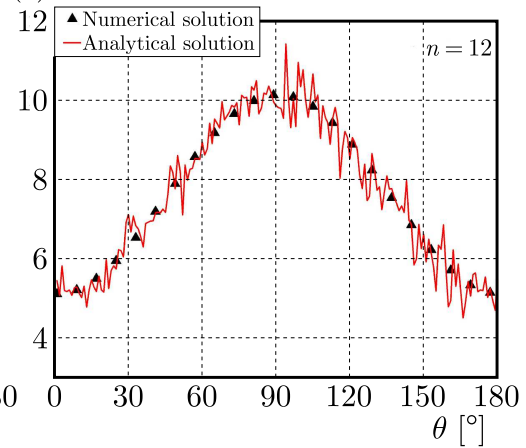

Fig. 8. Tangential stress contrast between the analytical solution obtained by the traditional Cauchy integral method and the numerical solution for different $n$ when $\rho=8$ : (a) $n=3$, (b) $n=6$, (c) $n=12$

\subsection{Improvement of the Cauchy integral method}

$\varphi_{0}(\zeta)$ obtained by the traditional Cauchy integral method is still used, but to ensure the reliability of calculation results, it is necessary to deal with $\psi_{0}(\zeta)$.

To make $\psi_{0}(\zeta)$ exclude the positive power term of $\zeta$, in this paper, Eq. (2.17) obtained by the traditional Cauchy integral solution method is not used to the calculate stress and displacement. Instead, Eq. (2.12) is used to solve $S_{k}^{\prime}$ in Eq. $(2.15)_{2}$, then $\psi_{0}(\zeta)$ does exclude the positive power term of $\zeta$. To determine $S_{k}^{\prime}$, the expression of $L_{k}^{\prime}$ in (2.9) must also be solved. It is obtained from Eq. $(2.8)_{1}$ that

$$
\begin{aligned}
L_{0}^{\prime} & =C_{0}+\sum_{j=1}^{n-1} j C_{j} L_{j+1} \quad L_{1}^{\prime}=1+\sum_{j=1}^{n} j C_{j} L_{j} \\
L_{k}^{\prime} & =\sum_{j=1}^{k-1} j C_{j} L_{k-j-1}^{\prime}+\sum_{j=1}^{n-k+1}(k-1+j) C_{k-1+j} L_{j} \quad k=2, \ldots, n \\
L_{k}^{\prime} & =\sum_{j=1}^{n} j C_{j} L_{k-j-1}^{\prime} \quad k=n+1, \ldots, \infty
\end{aligned}
$$

Substituting the boundary values of Eq. (2.9) and the obtained $\psi_{0}(\zeta)$ into Eq. (2.12), and comparing the coefficients of the same powers of $\sigma$, we have

$$
\begin{aligned}
S_{0}^{\prime} & =-\sum_{j=1}^{n-1} j \bar{a}_{j} L_{j+1} \quad S_{1}^{\prime}=-\sum_{j=1}^{n} j \bar{a}_{j} L_{j} \\
S_{k}^{\prime} & =-\sum_{j=1}^{n-k+1}(k-1+j) \bar{a}_{k-1+j} L_{j}-\sum_{j=1}^{k-1} j \bar{a}_{j} L_{k-1+j}^{\prime} \quad k=2, \ldots, n \\
S_{k}^{\prime} & =-\sum_{j=1}^{n} j \bar{a}_{j} L_{k-1+j}^{\prime} \quad k=n+1, \ldots, \infty
\end{aligned}
$$

Theoretically, the value range of $k$ in $S_{k}^{\prime}$ is $0 \sim \infty$, however, $k$ must be taken as a finite value in the actual calculation. Since $-\sum_{k=0}^{\infty} S_{k}^{\prime} \zeta^{-k}$ is convergent, the satisfactory results can be obtained when the number of terms of $S_{k}^{\prime}$ is high.

It can be obtained from Eqs. $(2.8)_{2}$ and $(2.15)_{2}$ that

$$
\psi_{0}(\zeta)=-\sum_{k=0}^{m} S_{k}^{\prime} \zeta^{-k}-\frac{P R}{2}(1+\lambda) \zeta^{-1}+\frac{P R}{2}(1-\lambda) \sum_{j=1}^{n} C_{j} \zeta^{-j}
$$


Substituting Eq. (2.1) and the obtained $\varphi_{0}(\zeta)$ into Eq. $(2.3)_{1}$ yields

$$
\varphi(\zeta)=\frac{P R}{4}(1+\lambda)\left(\zeta+\sum_{k=0}^{n} C_{k} \zeta^{-k}\right)+\sum_{j=1}^{n} a_{j} \zeta^{-j}
$$

Substituting Eqs. (2.1) and (3.3) into Eq. (2.3) 2 yields

$$
\psi(\zeta)=-\sum_{k=0}^{m} S_{k}^{\prime} \zeta^{-k}-\frac{P R}{2}(1+\lambda) \zeta^{-1}+\frac{P R}{2}(\lambda-1)\left(\zeta+C_{0}\right)
$$

Finally, substituting the obtained $\varphi(\zeta)$ and $\psi(\zeta)$ into Eqs. $(2.2)_{1,2}$, the stress components $\sigma_{\rho}$, $\sigma_{\theta}$ and $\tau_{\rho \theta}$ are determined. Similarly, substituting the obtained $\varphi_{0}(\zeta)$ and $\psi_{0}(\zeta)$ into Eq. $(2.2)_{3}$, the displacement components $u$ and $v$ are found.

\subsection{The effects of the number of negative power terms in $\psi_{0}(\zeta)$ on the calculation results}

To study the effect of the number $m$ of negative power terms in $\psi_{0}(\zeta)$ on the calculation results of the improved method, a vertical-wall semicircle tunnel is taken as an example. The in-situ stress and parameters of the surrounding rock are the same as those in Section 2.5.

Figure 9 shows tangential stress contrast between analytical and numerical solutions for different $m$ when $\rho=1$. It can be seen from Fig. 9 that with an increase of $m$, the fluctuation of analytical solutions became smaller and calculation accuracy is higher, For the vertical-wall semicircle tunnel, when $m$ is equal to 200 , satisfactory results are obtained.

(a)

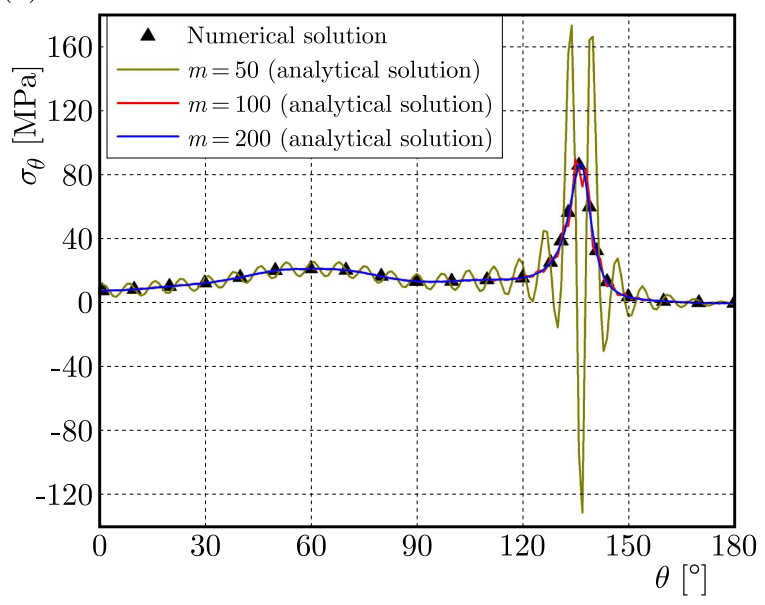

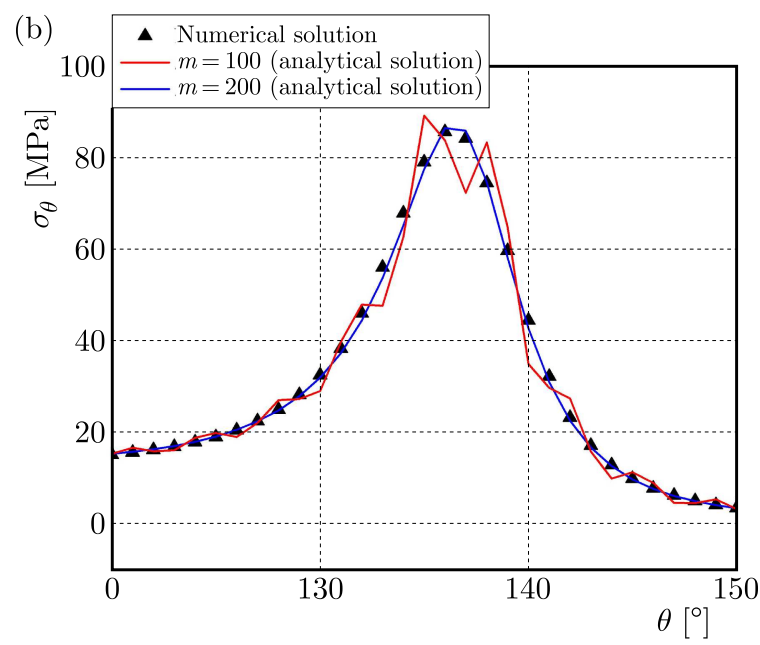

Fig. 9. Tangential stress contrast between the analytical solution obtained by the improved Cauchy integral method and the numerical solution for different $m$ when $\rho=1$ : (a) tangential stress, $0^{\circ} \leqslant \theta \leqslant 180^{\circ} ;(\mathrm{b})$ tangential stress, $120^{\circ} \leqslant \theta \leqslant 150^{\circ}$

\subsection{Validation of results}

The improved Cauchy integral method given in Section 3.3 is actually an analytical method, but its accuracy depends on the number $m$ of negative power terms in $\psi_{0}(\zeta)$. In the following, to verify the correctness of the improved Cauchy integral method, the improved Cauchy integral method is used to calculate the same example given in Section 2.5 (according to Section 3.3, $m=200$ ). The comparison of the improved analytical solution and numerical solutions of stress and displacement at $\rho=1, \rho=6$ and $\rho=8$ are given in Figs. 10-12, respectively. It can be seen from Figs. 10-12 that: 
The improved analytical solutions of stress and displacement at $\rho=1, \rho=6$ and $\rho=8$ are basically the same as the numerical solutions, which verifies the correctness of the improved method in this paper.

Through the improved method, the distribution of stress and displacement outside the tunnel can be obtained with high accuracy, and the problem of abnormal calculation will not appear.

(a)

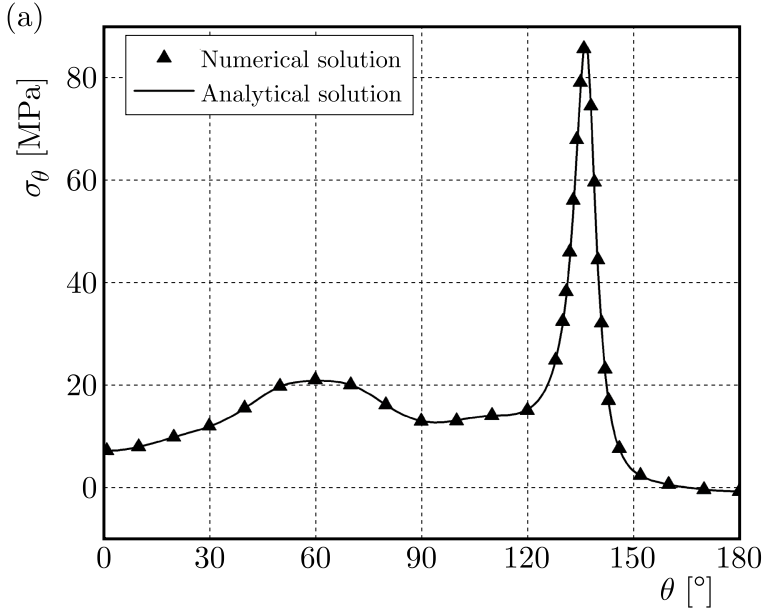

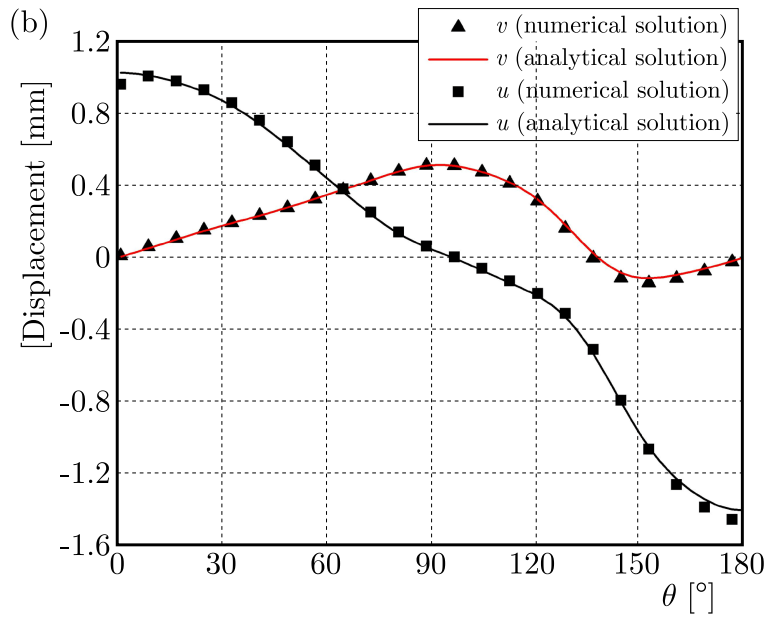

Fig. 10. The contrast between analytical solution obtained by improved Cauchy integral method and numerical solution when $\rho=1$ : (a) comparison of tangential stress, (b) comparison of displacement

(a)

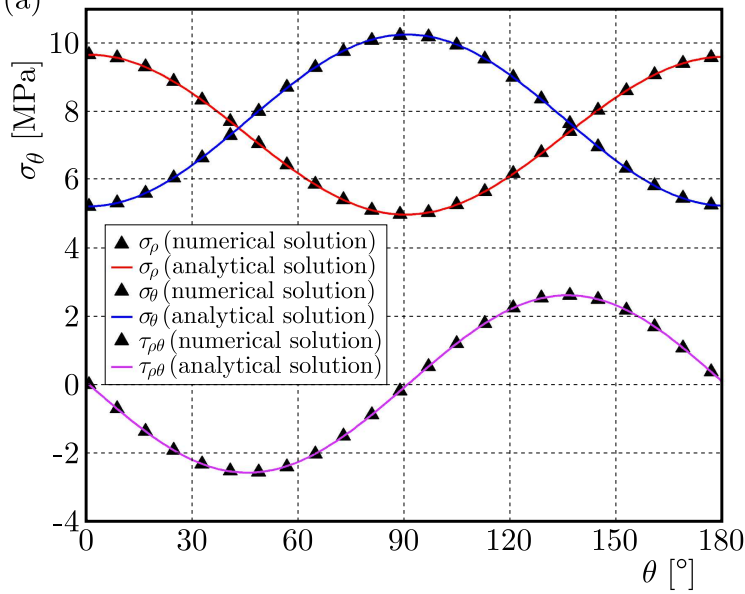

(b)

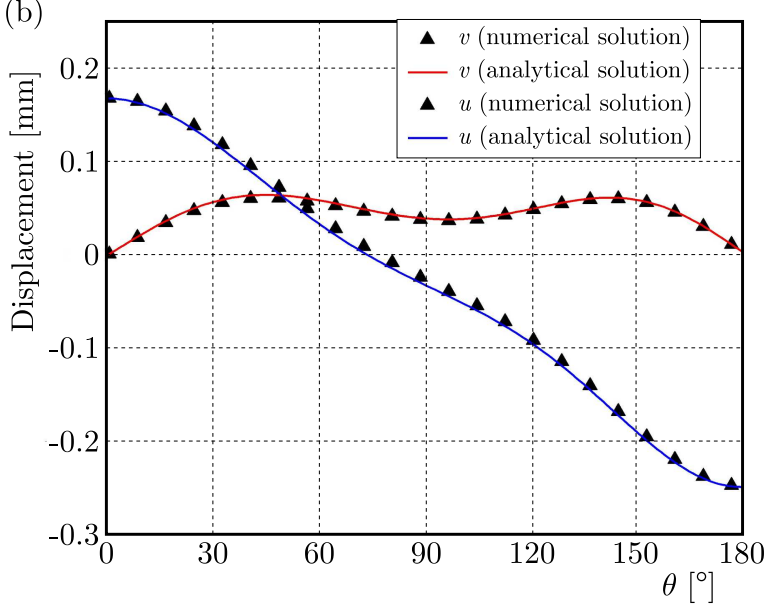

Fig. 11. The contrast between the analytical solution obtained by the improved Cauchy integral method and the numerical solution when $\rho=6$ : (a) comparison of stress, (b) comparison of displacement

\section{Conclusion}

For a simple-shaped tunnel with the number of mapping function items $n$ less than 3 , such as a circle, an ellipse, etc., accurate stress and displacement solutions can be obtained at the edge of the tunnel and the area outside the tunnel by the traditional Cauchy integral method. For a complex-shaped tunnel with $n \geqslant 3$, accurate solutions can still be obtained at the edge of the tunnel. However, due to the rounding error caused by the computer operation, there are positive power terms of $\zeta$ in $\psi_{0}(\zeta)$. The positive power of $\zeta$ is higher with the increasing $n$, and the value of $|\zeta|(\rho)$ is larger as the distance from the calculated position to the tunnel edge is increased. Therefore, there are some positive high powers of large values in the actual 
(a)

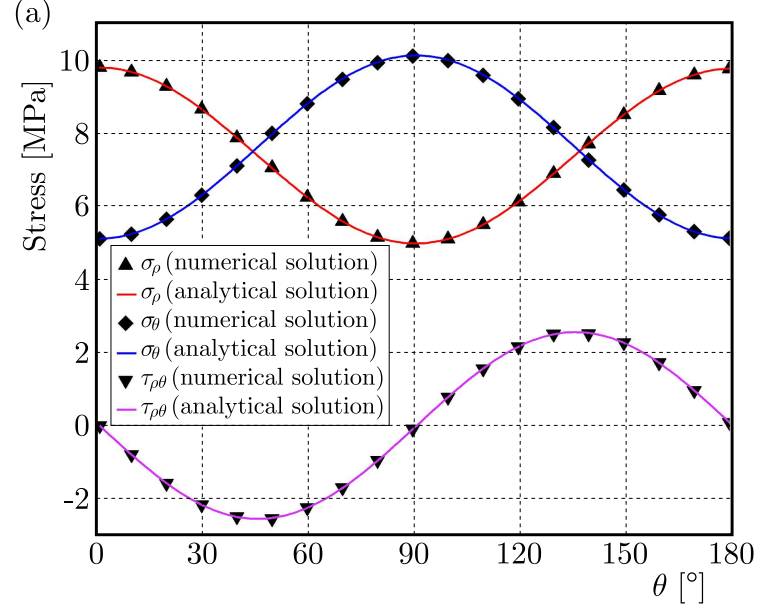

(b)

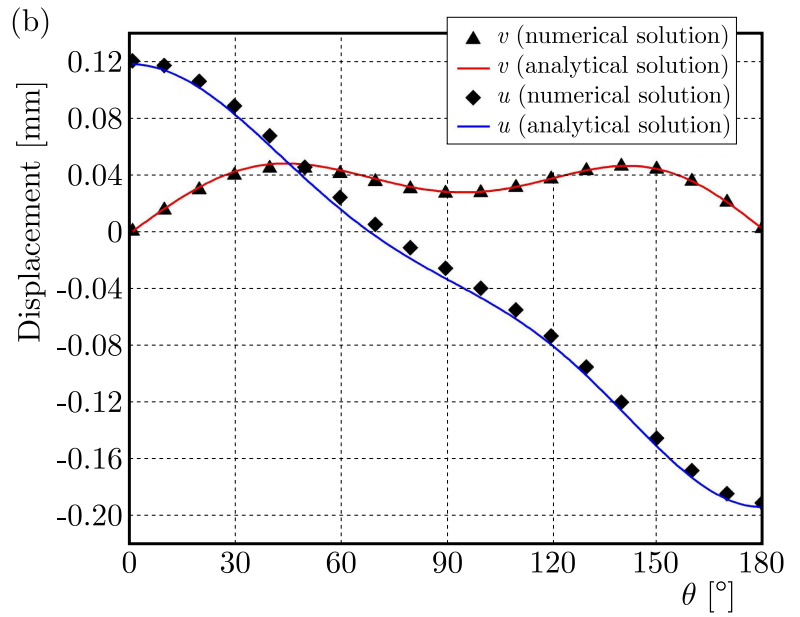

Fig. 12. The contrast between the analytical solution obtained by the improved Cauchy integral method and the numerical solution when $\rho=8$ : (a) comparison of stress, (b) comparison of displacement

calculation, which leads to a large calculation error and abnormal calculation. The calculated stress and displacement produce a very unstable oscillation, i.e., the stress and displacement calculated by the complex potential function obtained by the traditional Cauchy integral method are abnormal.

In this paper, $\psi_{0}(\zeta)$ is improved so that the expression of $\psi_{0}(\zeta)$ only contained negative power terms of $\zeta$ and a constant term. When the number of negative power terms is $m$, the expressions of negative power terms and constant terms are obtained by the undetermined coefficient method. It is found from the calculation example that the calculation accuracy of stress and displacement is higher with the increasing of $m$. For the vertical-wall semicircle tunnel, when $m$ is equal to 200, satisfactory results are obtained. The improved $\psi_{0}(\zeta)$ is applied to the calculation of stress and displacement in the surrounding rock, which eliminates abnormal calculation of the stress and displacement outside the tunnel. The analytical method of the stress and displacement around the deeply-buried tunnel is established, which is suitable for the edge of the tunnel and the area outside the tunnel.

\section{Acknowledgments}

Funding: This work was supported by the National Natural Science Foundation of China (grant number 51974124).

\section{Reference}

1. Duncan J.M., Dunlop P., 1968, Slopes in stiff-fissured clays and shales, ASCE Soil Mechanics and Foundation Division Journal, 95, 467-492

2. Exadaktylos G.E., Stavropoulou M.C., 2002, A closed-form elastic solution for stresses and displacements around tunnels, International Journal of Rock Mechanics and Mining Sciences, 39, 905-916

3. Fakhimi A., Salehi D., Mojtabai N., 2004, Numerical back analysis for estimation of soil parameters in the Resalat Tunnel project, Tunnelling and Underground Space Technology, 19, $57-67$

4. Greenspan M., 1944, Effect of a small hole on the stresses in a uniformly loaded plate, Quarterly of Applied Mathematics, 2, 60-71

5. InGLis C.E., 1913, Stresses in a plate due to the presence of cracks and sharp corners, Transactions of the Royal Institution of Naval Architects, 55, 219-241 
6. Jiao C.M., Zhao C.F., Lou Y., Lu A.Z., Shi Z.M., 2009, Research on stochastic back analysis using of Cauchy integral method and genetic algorithm, Rock and Soil Mechanics, 30, 251-256

7. Kodama J., Miyamoto T., Kawasaki S., Fujin Y., Kaneko K., Hagan P., 2013, Estimation of regional stress state and Young's modulus by back analysis of mining-induced deformation, International Journal of Rock Mechanics and Mining Sciences, 63, 1-11

8. Lei G.H., NG C., Rigby D.B., 2001, Stress and displacement around an elastic artificial rectangular hole, Journal of Engineering Mechanics, 127, 880-890

9. Lu A.Z., Zhang L.Q., 2007, Complex Variable Method for Mechanical Analysis of Underground Tunnel (in Chinese), Science Press, Beijing

10. Моток M.D., 1997, Stress concentration on the contour of a plate opening of an arbitrary corner radius of curvature, Marine Structures, 10, 1-12

11. Muskhelishvili N.I., 1963, Some Basic Problems of the Mathematical Theory of Elasticity, Noordhoff, Groningen

12. Sauers T., 2012, Numerical Analysis, 2nd ed., Pearson, New York

13. Sharma D.S., 2012, Stress distribution around polygonal holes, International Journal of Mechanical Sciences, 65, 115-124

14. Sharma D.S., 2016, Stresses around hypotrochoidal hole in infinite isotropic plate, International Journal of Mechanical Sciences, 105, 32-40

15. Shi Y.Z., Gao X.N., 2014, Complex variable function solution of stress and displacement of surrounding rock buried deep horseshoe-shaped tunnel excavation, Journal of Civil, Architectural and Environmental Engineering, 36, 101-118

16. Terzaghi K., Richart F.E., 1952, Stresses in rock about cavities, Géotechnique, 3, 57-90

17. ZhaO G.P., YANG S.L., 2015, Analytical solutions for rock stress around square tunnels using complex variable theory, International Journal of Rock Mechanics and Mining Sciences, 80, 302-307 